\title{
Study on the application of ultrasonography in the diagnosis of fetal cardiac structural abnormalities and the relationship between fetal cardiac structural abnormalities with chromosome abnormalities in early pregnancy
}

\author{
Xueqin Ji ${ }^{1,2}$, Yan $\mathrm{Xia}^{2}$, Hao Zhang ${ }^{2}$, Hairui Wang ${ }^{3}$, Ruixian $\mathrm{Shi}^{4}$, Zhen $\mathrm{Han}^{1}$ \\ ${ }^{1}$ Department of Obstetrics and Gynecology, The First Affiliated Hospital of Xi'an Jiao Tong University, Xi'an, China; ${ }^{2}$ Department of Ultrasound \\ Medicine, Ningxia Maternal and Child Health Care Hospital (Ningxia Children's Hospital), Yinchuan, China; ${ }^{3}$ Ningxia Medical University, \\ Yinchuan, China; ${ }^{4}$ Department of Prenatal Diagnosis Center, the Center of General Hospital of Ningxia Medical University, Yinchuan, China \\ Contributions: (I) Conception and design: Z Han, X Ji, H Zhang; (II) Administrative support: Z Han; (III) Provision of study materials or patients: X \\ Ji, Y Xia, H Wang, R Shi; (IV) Collection and assembly of data: X Ji, Y Xia, H Wang, R Shi; (V) Data analysis and interpretation: H Zhang, Y Xia; (VI) \\ Manuscript writing: All authors; (VII) Final approval of manuscript: All authors. \\ Correspondence to: Zhen Han, MD. The Department of Obstetrics and Gynecology, The First Affiliated Hospital of Xi'an Jiao Tong University, No. \\ 277 Yanta West Road, Xi'an 710061, China. Email: hanamy02@163.com.
}

Background: To explore the significance of multiple ultrasonic soft indexes such as Nuchal translucency (NT) in detection of cardiac structural malformations and chromosome abnormalities in fetal systematic screening in the first trimester, and to understand the value of combined transvaginal ultrasound (TVUS) in congenital heart disease (CHD) screening.

Methods: A total of 3,356 pregnant women who underwent early NT screening were screened by systematic ultrasound to monitor and evaluate the sensitivity and specificity of NT, tricuspid valve (TV), ductus venosus (DV) in the diagnosis of fetal CHD. According to the different intervals of NT thickening, the patients were divided into four groups, the detection rates of CHD and abnormal karyotypes in each group were compared, and the consistency of transabdominal and combined transvaginal ultrasonography was compared.

Results: A total of 3,356 cases of early pregnancy were examined by NT. A total of 66 cases of CHD were detected, and the detection rate was $1.97 \%$. Among the 66 CHD cases, 14 cases underwent chromosome karyotype examination and 12 of those cases had abnormal results. With the increase of NT thickness, the detection rates of cardiac structural abnormalities and chromosomal abnormalities all showed a linear increasing trend. The sensitivity of the NT $\geq 2.5 \mathrm{~mm}$ group was as high as $63.64 \%$, and the ductus venosus $\alpha$ wave (DV $\alpha$ ) reverse specificity, and the positive likelihood ratio was $99.57 \%$ and $53.41 \%$, respectively. The sensitivity of the 3 indicators combined was $66.67 \%$, which was higher than that of any single index, and the area under the receiver operating characteristic (ROC) curve of these 3 indicators combined was the largest (AUC: 0.86). Seventy patients in total were examined by combined TVUS. There is no statistical difference between the two.

Conclusions: A positive linear correlation was found between NT thickness and the detection rate of fetal cardiac structural abnormality and chromosome abnormality. Early pregnancy NT screening combined with TV blood flow spectrum and DV blood spectrum screenings has high specificity and sensitivity in the diagnosis of CHD. Combined transabdominal and TVUS in early pregnancy can reduce the rates of misdiagnosis and missed diagnosis of fetal CHD.

Keywords: Early pregnancy; congenital heart disease (CHD); chromosome; vaginal ultrasound

Submitted Nov 05, 2021. Accepted for publication Dec 17, 2021.

doi: 10.21037/atm-21-6492

View this article at: https://dx.doi.org/10.21037/atm-21-6492 


\section{Introduction}

Congenital heart disease (CHD) is the most common serious congenital malformation in fetuses. $25 \%$ of CHD children die in childhood after birth, accounting for more than half of the cases of fatal congenital malformations in childhood (1), and CHD is also the most expensive treatment of birth defects (2). CHD is usually caused by environmental factors, genetic factors alone or both, of which $75-90 \%$ are caused by environmental and genetic factors (3). Therefore, early fetal screening, diagnosis, and early intervention are very important if the rates of fetal and childbirth malformations and mortality are to be reduced. The risk of CHD is closely related to the type of fetal heart malformation, and multiple studies have found that the incidence of CHD in fetal chromosomal karyotype abnormalities is as high as $40 \%$ (4-6).

Studies have shown that the incidence of fetal heart anatomical structure abnormalities with non-immune effusion in vivo is $10-20 \%(7,8)$. The measurement of nuchal translucency (NT) thickness during early pregnancy (11-14 weeks) is an effective method for assessing the risk of fetal chromosomal abnormalities. Many studies have suggested that an increase in NT thickness is related to most fetal malformations (including heart malformations) and genetic syndromes $(9,10)$, and the risk of CHD is positively correlated with an increase in NT thickness (11). In addition, a number of studies have shown that soft indicators, such as tricuspid regurgitation, and the a-wave reversal of ductus arteriosus in early pregnancy also contribute to the detection rate of CHD (12-14). Thus, it is necessary to perform NT and fetal heart structure examinations at the same time during early pregnancy.

Because of the small size of the fetus in early pregnancy, the examination of CHD is more difficult. Research suggests that in early pregnancy, a transvaginal ultrasound (TVUS) can obtain higher-resolution image quality than an abdominal ultrasound, and some scholars contend that TVUS clearly shows cardiac structure in fetuses with a fetal crown rump length (CRL) of 45-84 $\mathrm{mm}$, or as early as $11+$ weeks of gestation. However, TVUS has certain requirements related to the fetal position and operator skill level, and TVUS cannot be widely used due to the limitation of the probe angle.

In this study, transabdominal or combined transvaginal ultrasonography was used in early pregnancy to study the value of NT and other ultrasound soft indexes in the detection of fetal cardiac structural malformations and chromosome abnormalities in early pregnancy through systematic screening.

We present the following article in accordance with the STARD reporting checklist (available at https://dx.doi. org/10.21037/atm-21-6492).

\section{Methods}

\section{General information}

The data of 3,356 women with singleton or twin pregnancies who underwent fetal NT examinations in early pregnancy (gestation: 11-14 weeks; CRL: 45-84 mm) or grade II or III structural screening examinations in early pregnancy (gestation: 16-18 weeks) at the General Hospital of Ningxia Medical University from January 2017 to December 2018 were collected. NT thickening was defined as NT $>2.5 \mathrm{~mm}$ (11-14 weeks) or cystic tumor in fetal neck were defined as NT thickening. The fetal CRL at 11-14 weeks of gestation was $54-84 \mathrm{~mm}$. The pregnant women who received standard prenatal ultrasound scanning at our hospital in the first trimester or the first trimester of pregnancy were taken as the subjects of study. Operators who measure NT need to obtain British Fetal Medicine Foundation (FMF) qualification certification and receive standardized prenatal screening training.

Diagnostic criteria: in line with the British FMF NT measurement standards; in line with the International Association of Obstetrics and Gynecology (ISUOG) in 2013 issued by the International Association of Obstetrics and Gynecology ultrasound guidelines for early pregnancy fetal ultrasound screening guidelines (15) and the latest fetal heart ultrasound screening practice guide (16) provided in the screening section and heart parameters. NT thickening was defined as NT $>2.5 \mathrm{~mm}$ (11-14 weeks) or cystic tumor in the fetal neck. Chromosomal abnormalities are defined as chromosomal nucleus type abnormalities diagnosed by amniocentesis punctures.

All procedures performed in this study involving human participants were in accordance with the Declaration of Helsinki (as revised in 2013). The study was approved by Ningxia Medical University General Hospital scientific research Ethics Committee and informed consent was taken from all the mothers.

\section{Instrument}

The ultrasonic diagnostic instrument Voluson 8 (GE 

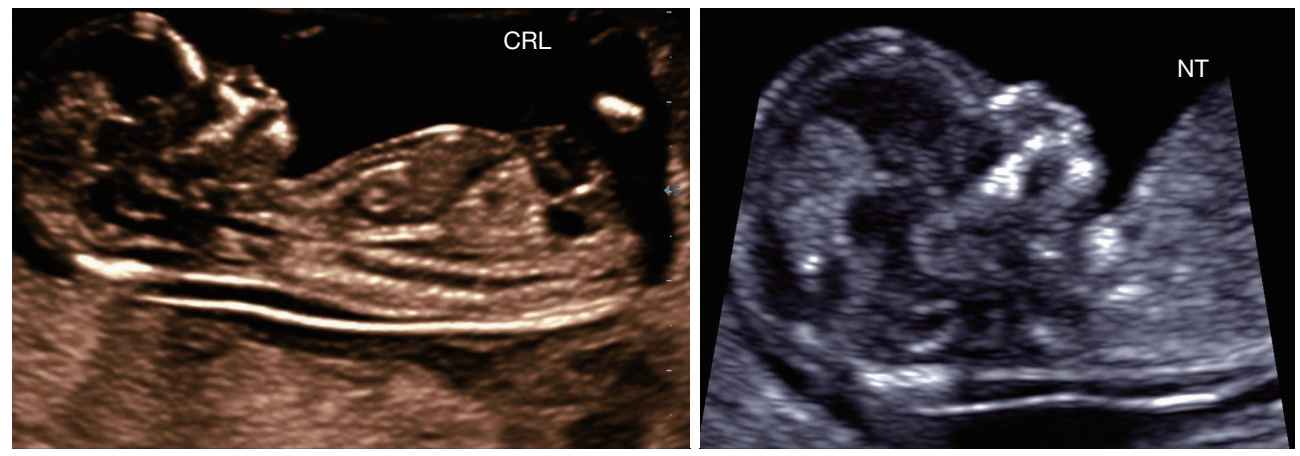

Figure 1 CRL and NT standard measurement images. CRL, crown rump length; NT, nuchal translucency.

Company) was used, which was equipped with a linear array probe for the transabdominal ultrasound and TVUS, the three-dimensional volume probe for fetal echocardiography, and spatial-temporal image correlation (STIC) technical software. It has the functions of local amplification, tissue harmonic imaging, movie playback, and dynamic storage. The power is $<100 \mathrm{MW} / \mathrm{cm}^{2}$. It can automatically optimize an image. The frequency of the abdominal linear array probe is $2-8 \mathrm{MHz}$, the frequency of the transvaginal linear array probe is $4-9 \mathrm{MHz}$, and the frequency of the abdominal volume probe is 4-8 MHZ.

The images collected were as follows: CRL measurement map in early pregnancy (Figure 1), NT measurement map in early pregnancy (Figure 1), venous catheter blood flow spectrum in early and middle pregnancy, tricuspid flow spectrum in early and middle pregnancy. The static and dynamic pictures of the abnormal part of the fetal structure were stored. Fetuses with a CRL of $45-84 \mathrm{~mm}$ were systematically screened in early pregnancy. NT, venous catheter, tricuspid valve blood flow, and other ultrasound soft indexes were also detected and recorded. If necessary, TVUS was used to screen fetal heart structure. All fetuses suspected of cardiac structural abnormalities in early pregnancy were diagnosed by 1-2 fetal prenatal ultrasound diagnostic experts and suggested that pregnant women should be screened at II or III level in the second trimester (18-22 weeks), including echocardiography examination, then compared with the results of early pregnancy examination. Abnormal cases were examined by chromosome karyotype based on informed consent, followed up the results of prenatal diagnosis, and fetal autopsy was performed after informed consent was selected for termination of pregnancy. The newborn fetus was followed up and the physical examination of the newborn was carried out.

\section{Statistical analysis}

SPSS 24.0 was used. The counting data are expressed by the rate. If the measurement data conformed to a normal distribution, the mean \pm standard deviation is used, if not, the median (interquartile range) is used. The comparison of the detection rate of CHD by transabdominal and transvaginal ultrasound in early pregnancy was carried out by chi-square test with a paired design of four-grid table. The sensitivity, specificity, positive predictive value, negative predictive value, and positive likelihood ratio of these diagnostic tests were compared to evaluate the prediction accuracy of CHD. The test level was $\alpha=0.05$.

\section{Results}

\section{CHD diagnosis and prenatal diagnosis results}

A total of 3,356 pregnant women with an average age of 29.18 \pm 4.55 underwent NT examinations. All these 3,356 pregnant women can show the NT, tricuspid valve, and venous catheter. The average value of NT was $1.62 \pm$ $0.83 \mathrm{~mm}$, and the average CRL was $6.62 \pm 0.89 \mathrm{~cm}$. There were 172 cases of NT thickening, including 42 cases of fetal heart structure abnormality. Twenty-one cases were scanned with a transabdominal combined TVUS. A total of 3,184 cases of normal NT values were associated with CHD 24 cases. Eleven cases were scanned by a transabdominal combined TVUS, and 66 cases of CHD were detected; the detection rate was $1.97 \%$. There were 33 cases of induced labor in early pregnancy, and 33 cases in the second trimester by systematic screening and echocardiography. the diagnostic results were consistent with those in early pregnancy, including 32 cases of induced labor, 1 case of birth, a total of 65 cases of induced labor. Among the 66 cases of cardiac structural abnormality, 14 cases were 
Table 1 CHD examination and pregnancy outcome in 66 cases

\begin{tabular}{lccc}
\hline Items & NT thickening & NT normal & Total \\
\hline CHD was detected in early pregnancy & 42 & 24 & 11 \\
Combined transvaginal ultrasonography & 21 & 56 & 32 \\
Induced labor in early pregnancy & 28 & 19 & 18 \\
Reexamination in the second trimester & 14 & 53 & 32 \\
Induced labor in the second trimester & 9 & 5 & 14 \\
Chromosome examination & 8 & 4 \\
\hline
\end{tabular}

$\mathrm{CHD}$, congenital heart disease; NT, nuchal translucency.

Table 2 Abnormal karyotype distribution and fetal ultrasound findings in 12 cases

\begin{tabular}{lcl}
\hline Chromosomal karyotype & Number of cases & Ultrasound performance \\
\hline $47, \mathrm{XN}+18$ & 6 & $\begin{array}{l}\text { NT thickness, holoprosencephaly, TOF, PLSVC, strawberry head, wide eye } \\
\text { distance, small mandible, single umbilical artery }\end{array}$ \\
$47, \mathrm{XN}+21$ & 4 & NT thickness, VSD, TR, incomplete ossification of nasal bone, PLSVC, CoA \\
$45, \mathrm{XN}$ & 2 & Cervical cystic tumor, VSD, tricuspid regurgitation
\end{tabular}

NT, nuchal translucency; VSD, ventricular septal defect; TR, tricuspid regurgitation; TOF, tetralogy of Fallot; CoA, coarctation of the aorta; PLSVC, perpetuate the left superior vena cava.

examined by chromosome karyotype (amniocentesis), and 12 cases were abnormal. Specifically, there were 6 cases of trisomy-18 syndrome, 4 cases of trisomy-21 syndrome and 2 cases of Turner syndrome. In early pregnancy, 8 cases were diagnosed as CHD by abdominal ultrasound, including 3 cases of tetralogy of Fallot (TOF) missed diagnoses, 1 case of persistent left superior vena cava (PLSVC), 1 case of pulmonary stenosis, 1 case of an abnormal origin of the right pulmonary artery, 1 case of coarctation of the aorta arch (CoA), 1 case of coarctation of the aortic arch with aberration of right subclavian artery (ARSA). Among the 8 missed cases, 6 cases were examined by chromosome karyotype in the second trimester, including 1 case of trisomy-18 syndrome, 1 case of trisomy-21 syndrome and 4 cases of normal karyotypes. Among these 8 missed cases, 1 case of birth and 7 cases of induced labor (Tables 1-3, Figure 2).

Fifteen cases were autopsied; 12 cases of fetal autopsy in early pregnancy were consistent with the ultrasound diagnoses; 3 cases of fetal autopsy in the second trimester were missed diagnoses in early pregnancy, which were diagnosed as abnormal origin of right pulmonary artery (RPA), CoA and TOF by echocardiography in the second trimester. The results of autopsy were consistent with the ultrasonic diagnosis of the second trimester.

\section{The correlation between NT thickening and the detection of cardiac malformation and abnormal chromosome}

Ninety-eight cases of 172 fetuses with NT thickening underwent chromosome examinations, and 8 of 9 fetuses with cardiac malformation had chromosomal abnormalities. The fetuses of with NT thickening were divided into 4 groups according to the NT thickening interval, and the detection rates of cardiac malformation and chromosomal abnormality in different thicknesses were compared (see Table 4). When the NT thickness was $>5.5 \mathrm{~mm}$, the incidence of fetal combined cardiac structural abnormality was nearly 17 times of that in the $2.5-3.4 \mathrm{~mm}$ interval group, while the detection rate of chromosomal abnormality was nearly 28 times of that in the $2.5-3.4 \mathrm{~mm}$ interval group (see Figure 3). The trend chi-square test of the 4 interval groups was statistically significant $(\mathrm{P}<0.05)$; that is, with the increase of the thickness of NT, the incidence of cardiac structural abnormalities and the detection rate of chromosomal abnormalities increased linearly. 
Table 3 Missed cases and pregnancy outcomes

\begin{tabular}{|c|c|c|c|c|c|c|}
\hline $\begin{array}{l}\text { Cases } \\
\text { number }\end{array}$ & $\begin{array}{l}\text { Initial check } \\
\text { week }\end{array}$ & $\begin{array}{l}\text { Recheck } \\
\text { week }\end{array}$ & $\begin{array}{c}\text { Initial } \\
\text { diagnosis }\end{array}$ & Recheck diagnosis & $\begin{array}{c}\text { Chromosomal karyotype } \\
\text { examination }\end{array}$ & Pregnancy outcome \\
\hline 1 & 13 & 18 & Normal & TOF & Not done & Induced labor \\
\hline 3 & 13 & 18 & Normal & TOF & $47, \mathrm{XN}+18$ & Induced labor \\
\hline 6 & 13 & 18 & TR & PS, TR & $46, \mathrm{XN}$ & Induced labor \\
\hline 7 & 13 & 18 & NT thickness & PLSVC & $46, X N$ & Birth \\
\hline 8 & 13 & 19 & Normal & RPA originates from AAO & $46, \mathrm{XN}$ & Induced labor \\
\hline
\end{tabular}

NT, nuchal translucency; TR, tricuspid regurgitation; PS, pulmonary artery stenosis; RPA, right pulmonary artery; TOF, tetralogy of Fallot; AAO, ascending aorta; CoA, coarctation of the aorta; ARSA, aberration of right subclavian artery; PLSVC, perpetuate the left superior vena cava.
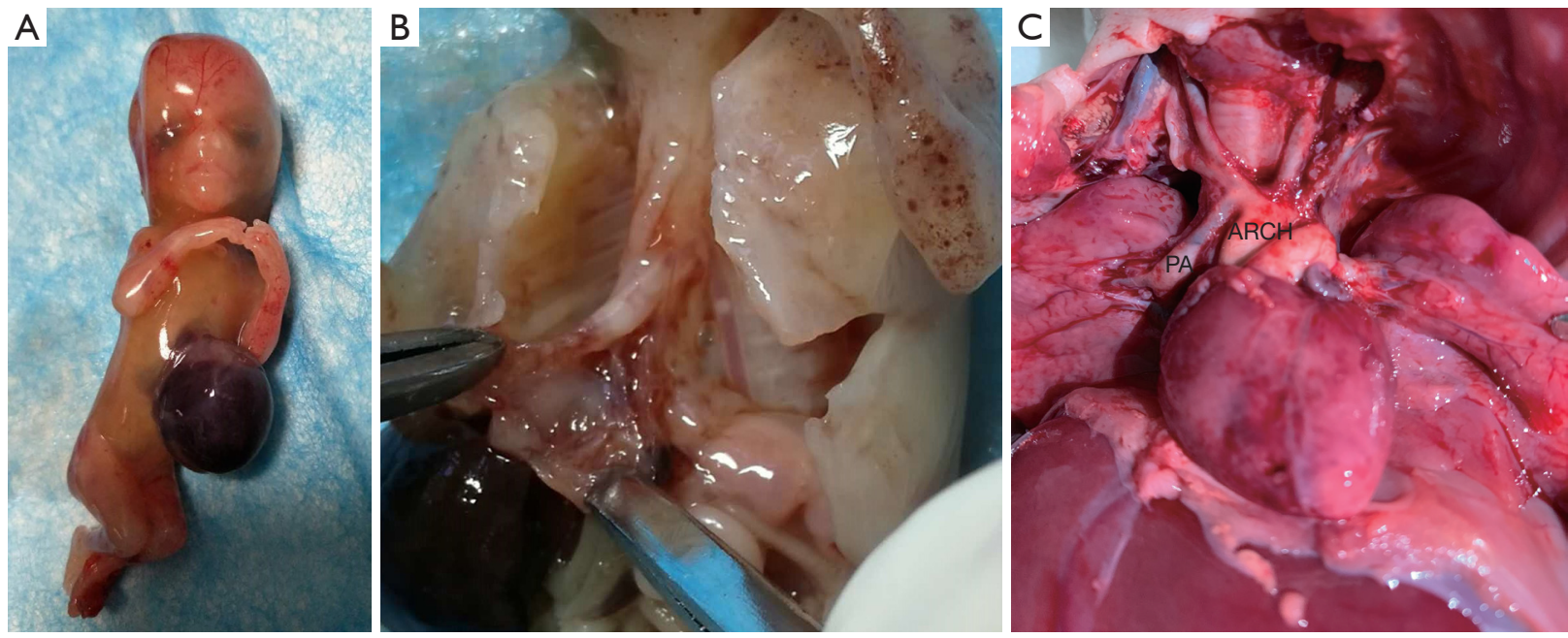

Figure 2 Autopsy photographs. (A) Omphalocele with cardiac malformation (12 weeks); (B) ventricular septal defect (12 weeks); (C) abnormal right pulmonary artery originating from the ascending aorta (22 weeks). These images are published with the consent from the infant's mother. PA, pulmonary artery; ARCH, aortic arch.

\section{The correlation between the soft index of ultrasounds and CHD in early pregnancy}

The NT screenings identified 172 cases of NT thickening, 55 cases of tricuspid regurgitation, and 29 cases of a-wave reversal of venous catheter. The predictive value of ultrasonic soft index to CHD showed that the sensitivity and negative predictive value of NT $\geq 2.5 \mathrm{~mm}$ group were higher than tricuspid regurgitation and venous catheter a wave reverse, which were $63.64 \%$ and $99.25 \%$; the reverse specificity, positive predictive value, and positive likelihood ratio of DV $\alpha$ wave were the highest, which were $99.57 \%$, $51.64 \%$ and 53.41 respectively.

In this study, 44 of 66 the CHD patients had at least 1 abnormal soft index, 4 of the 66 CHD patients had at least 3 abnormal soft indexes. As Table 5 shows, the sensitivity of combined 3 soft index screening is as high as $66.67 \%$, and the negative predictive value is $99.43 \%$. The data of the 3 soft index screening were higher than the sensitivity and negative predictive value of the single soft index screening, and the maximum area under the receiver operating characteristic (ROC) curve (AUC) can be obtained by 
Table 4 Comparison of the correlation between NT thickness and cardiac malformations and chromosomal abnormality

\begin{tabular}{lcccc}
\hline NT thickness $(\mathrm{mm})$ & Cases $(\mathrm{n}=172)$ & Heard malformation, $\mathrm{n}(\%)$ & Chromosomal karyotype & Abnormalities chromosomal, $\mathrm{n}(\%)$ \\
\hline $2.5-3.4$ & 111 & $6(5.41)$ & 68 & $2(2.9)$ \\
$3.5-4.4$ & 20 & $4(20.0)$ & 15 & $1(6.7)$ \\
$4.5-5.4$ & 17 & $10(58.8)$ & 3 & $1(33.3)$ \\
$>5.5$ & 24 & $22(91.7)$ & 5 & $4(80.0)$ \\
Trend & & 89.276 & & 29.912 \\
P & & 0.000 & 0.000 \\
\hline
\end{tabular}

NT, nuchal translucency.

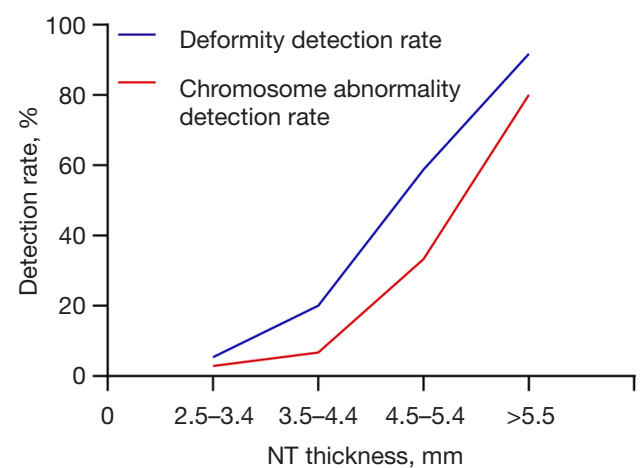

Figure 3 The relationship between NT thickness and detection rates of cardiac malformation or chromosomal abnormalities. NT, nuchal translucency.

combining the 3 (AUC $=0.861$; see Figure 4 and Table 5).

\section{Transabdominal and TVUS in early pregnancy}

Due to the factors such as small fetuses, a too thick fat layer in the pregnant women's abdominal walls, suspected cardiac structural abnormality, and unsatisfactory image quality of transabdominal ultrasound, a total of 70 cases were examined by combined TVUS in this study, and the smallest fetal CRL was $5.0 \mathrm{~mm}$. Among them, 32 cases had CHD and 38 cases did not have CHD. Among the 32 cases of CHD, the results of the combined transabdominal ultrasound and TVUS examination were consistent in 17 cases, and no obvious abnormality was found in 15 cases during the transabdominal ultrasound examination, and they were diagnosed as having a VSD, PLSVC and ARSA after TVUS examination and intra-department consultation. In the 38 negative cases, the abdominal and TVUS examination found that there were 27 cases of no significant cardiac structural abnormalities, 11 cases of suspected abdominal VSD, these were rule out the possibility of VSD after vaginal ultrasound examination, reviewing of echocardiography during pregnancy in the negative, and early pregnancy by TVUS results conform to (see Table 6, Figures 5,6).

Both a transabdominal and a combined transvaginal ultrasonography were performed on the same patient. The results are shown in Table 6. The paired chi-square test was used, and no difference was found between TVUS and transabdominal ultrasound $(\mathrm{P}=0.557)$.

\section{Discussion}

The thickening of NT is related to cardiac dysfunction caused by abnormal heart and large arteries, inherent problems in lymphoid development, or reduced lymphatic drainage caused by impaired fetal motor function (17). Gembruch and Geipel (18) found that the incidence of cardiac structural abnormalities increased in fetuses with thickened NT. This study is consistent with the study of Gembruch et al., the risk of chromosome abnormalities in NT thickening with multiple malformations is increased, which better reflects the correlation between NT thickening and fetal heart malformations and chromosomes.

DV is a high oxygen content blood that reaches the coronary artery and cerebral circulation through the umbilical vein and preferentially flows into the left atrium through the foramen ovale. The waveform change of DVa wave represents the pre- and post-load state of right atrial pressure, which is related to chromosome abnormalities, heart defects and adverse pregnancy outcome.

$\mathrm{TR}$ is common in right ventricular outflow tract obstructive CHD such as tricuspid dysplasia, Ebstein malformation, pulmonary stenosis (PS), pulmonary atresia with intact interventricular septum and contraction of 
Table 5 The forecast value of ultrasonic soft index to CHD

\begin{tabular}{|c|c|c|c|c|c|c|c|c|}
\hline $\begin{array}{l}\text { Ultrasonic soft } \\
\text { index }\end{array}$ & $\begin{array}{c}\text { Number } \\
\text { cases }\end{array}$ & $\mathrm{CHD}$ & $\begin{array}{l}\text { Normal } \\
\text { heart }\end{array}$ & $\begin{array}{c}\text { Sensitivity } \\
(\%)\end{array}$ & $\begin{array}{l}\text { Specificity } \\
(\%)\end{array}$ & $\begin{array}{c}\text { Positive prediction } \\
\text { value }(\%)\end{array}$ & $\begin{array}{c}\text { Negative prediction } \\
\text { value }(\%)\end{array}$ & $\begin{array}{c}\text { Positive like } \\
\text { hood ratio }\end{array}$ \\
\hline NT & & & & 63.64 & 96.05 & 24.36 & 99.25 & 16.10 \\
\hline$\geq 2.5 \mathrm{~mm}$ & 172 & 42 & 130 & & & & & \\
\hline$<2.5 \mathrm{~mm}$ & 3,184 & 24 & 3,160 & & & & & \\
\hline TR & 55 & 19 & 36 & & & & & \\
\hline Normal TV & 3,301 & 47 & 3,254 & & & & & \\
\hline Total & 3,356 & 66 & 3,290 & & & & & \\
\hline Total & 3,356 & 66 & 3,290 & & & & & \\
\hline Three soft indexes & & & & 66.67 & 94.83 & 17.34 & 99.43 & 12.9 \\
\hline $\begin{array}{l}\text { At least one is } \\
\text { abnormal }\end{array}$ & 14 & 44 & 170 & & & & & \\
\hline All are normal & 3,142 & 22 & 3,120 & & & & & \\
\hline Total & 3,356 & 66 & 3,290 & & & & & \\
\hline
\end{tabular}

CHD, congenital heart disease; NT, nuchal translucency; TV, tricuspid valve; TR, tricuspid regurgitation; DV $\alpha$, ductus venosus $\alpha$ wave.

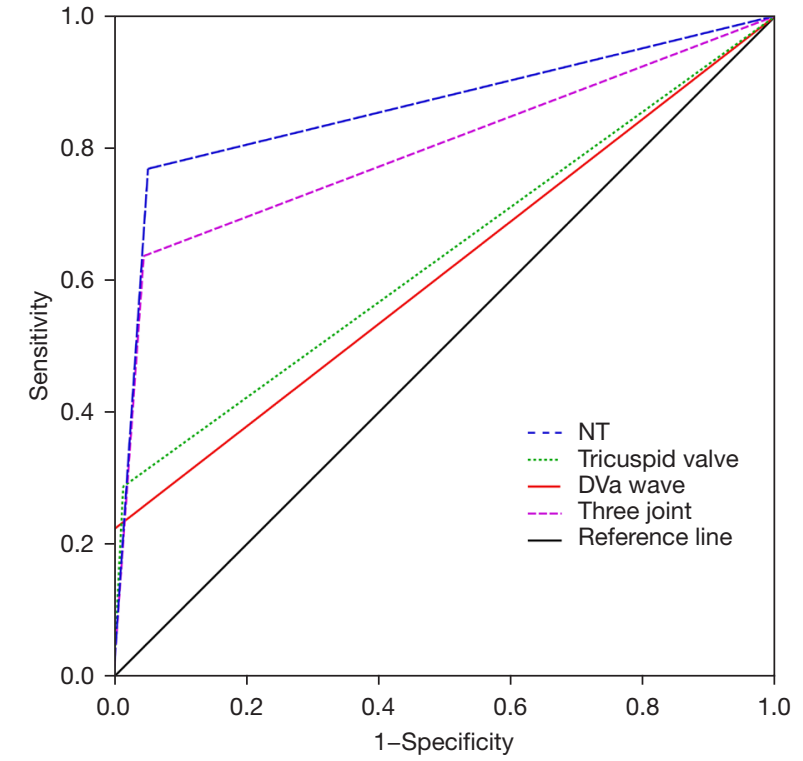

Figure 4 Area comparison results under the ROC curve of ultrasonic soft index. NT, nuchal translucency; DV $\alpha$, ductus venosus $\alpha$ wave; ROC, receiver operating characteristic.
Table 6 Comparison of consistency between transabdominal and combined TVUS

\begin{tabular}{lccc}
\hline \multirow{2}{*}{$\begin{array}{l}\text { Transabdominal } \\
\text { ultrasonography }\end{array}$} & \multicolumn{2}{c}{$\begin{array}{c}\text { Diagnosis of fetal heart by } \\
\text { combining TVUS }\end{array}$} & \multirow{2}{*}{ Total } \\
\cline { 2 - 3 } & Positive & Negative & \\
\hline Positive & 17 & 11 & 28 \\
Negative & 15 & 27 & 42 \\
Total & 32 & 38 & 70 \\
\hline
\end{tabular}

TVUS, transvaginal ultrasound .

ductus arteriosus, etc. Among the 55 cases of tricuspid regurgitation, 19 cases were complicated with CHD (the incidence rate was $34.5 \%$ ), with a specificity of $98.91 \%$, which was between NT thickening and DV $\alpha$ wave reverse, of which 16 cases were complicated with NT thickening.

In this study, the negative predictive values of NT greater than or equal to $2.5 \mathrm{~mm}$, the reverse a-wave of intravenous catheter and tricuspid regurgitation in the 3 

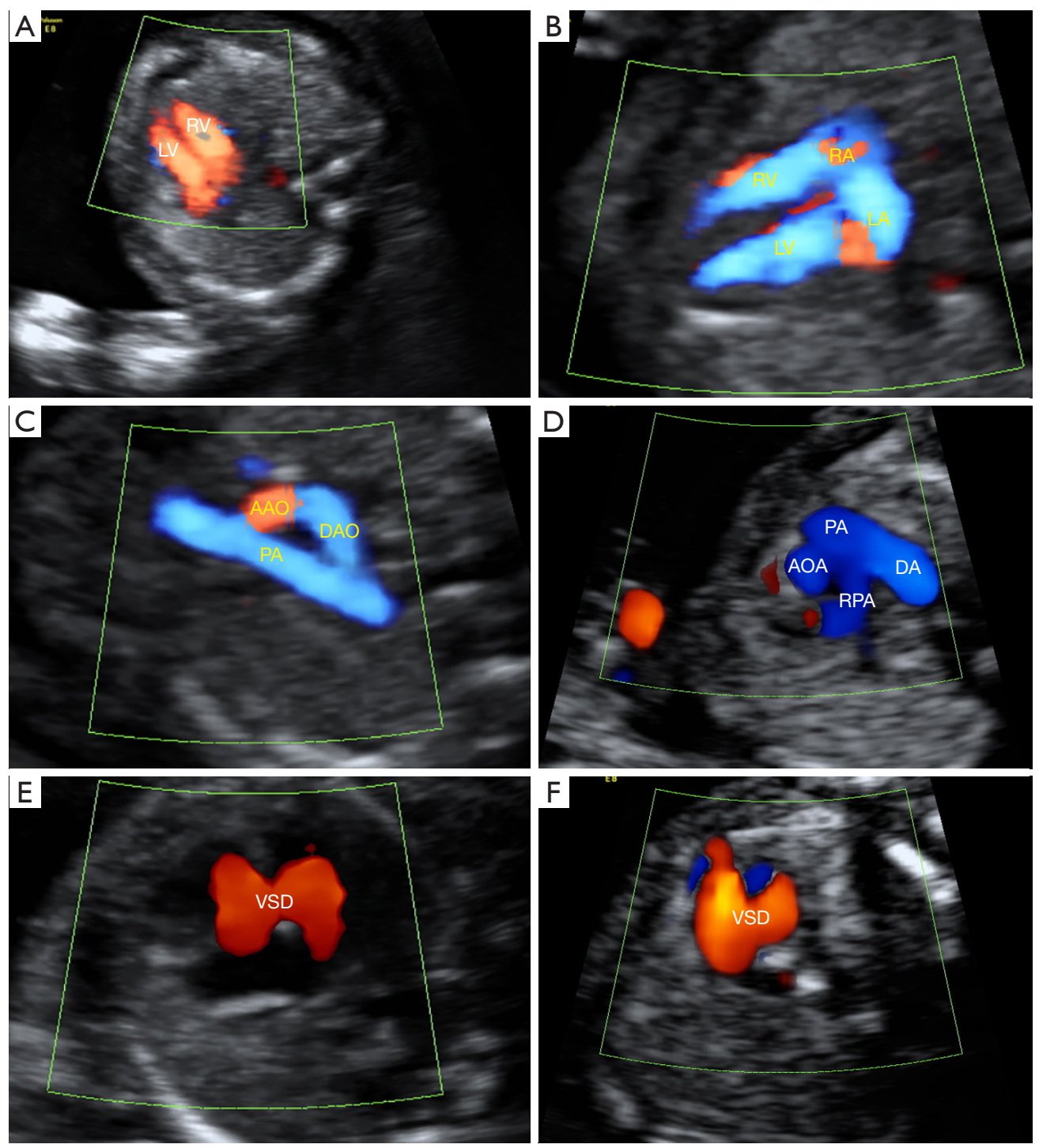

Figure 5 Comparison of resolution of transabdominal and TVUS images. (A-D) 12+ week-old fetus; (E,F) 11+ week-old fetus. (A) 4-chamber blood flow by transabdominal ultrasound; (B) 4-chamber blood flow by TVUS; (C) transabdominal ultrasound trivascular tracheal blood flow; (D) blood flow of 3 blood vessels by TVUS; (E) VSD by transabdominal ultrasound; (F) VSD by TVUS. TVUS, transvaginal ultrasound; VSD, ventricular septal defect; RV, right ventricle; LV, left ventricle; RA, right atrium; LA, left atrium; PA, pulmonary artery; RPA, right pulmonary artery; DA: arterial catheter; AOA, aorta; AAO, ascending aorta; DAO, descending aorta.

soft indicators of CHD screening were all higher than $98 \%$, and abnormal manifestations often suggested abnormal cardiac structure. The data of this study showed that the above 3 soft indicators had a very high predictive value for CHD. Further, the combination of the 3 indicators in early pregnancy fetal screening can significantly improve the sensitivity.

Abnormal body mass index led to the decline of transabdominal ultrasound image quality. In contrast, high-frequency transducers provide high-resolution image quality by shortening the distance between sound waves and the fetus. In this study, vaginal gynecological examination bed was used for transvaginal ultrasound examination for pregnant women, which expanded the scope of probe beam scanning, and improved the problem that the angle of transvaginal ultrasound scanning was too limited. The resolution of four-chamber heart and left and right ventricular outflow tract is significantly higher 

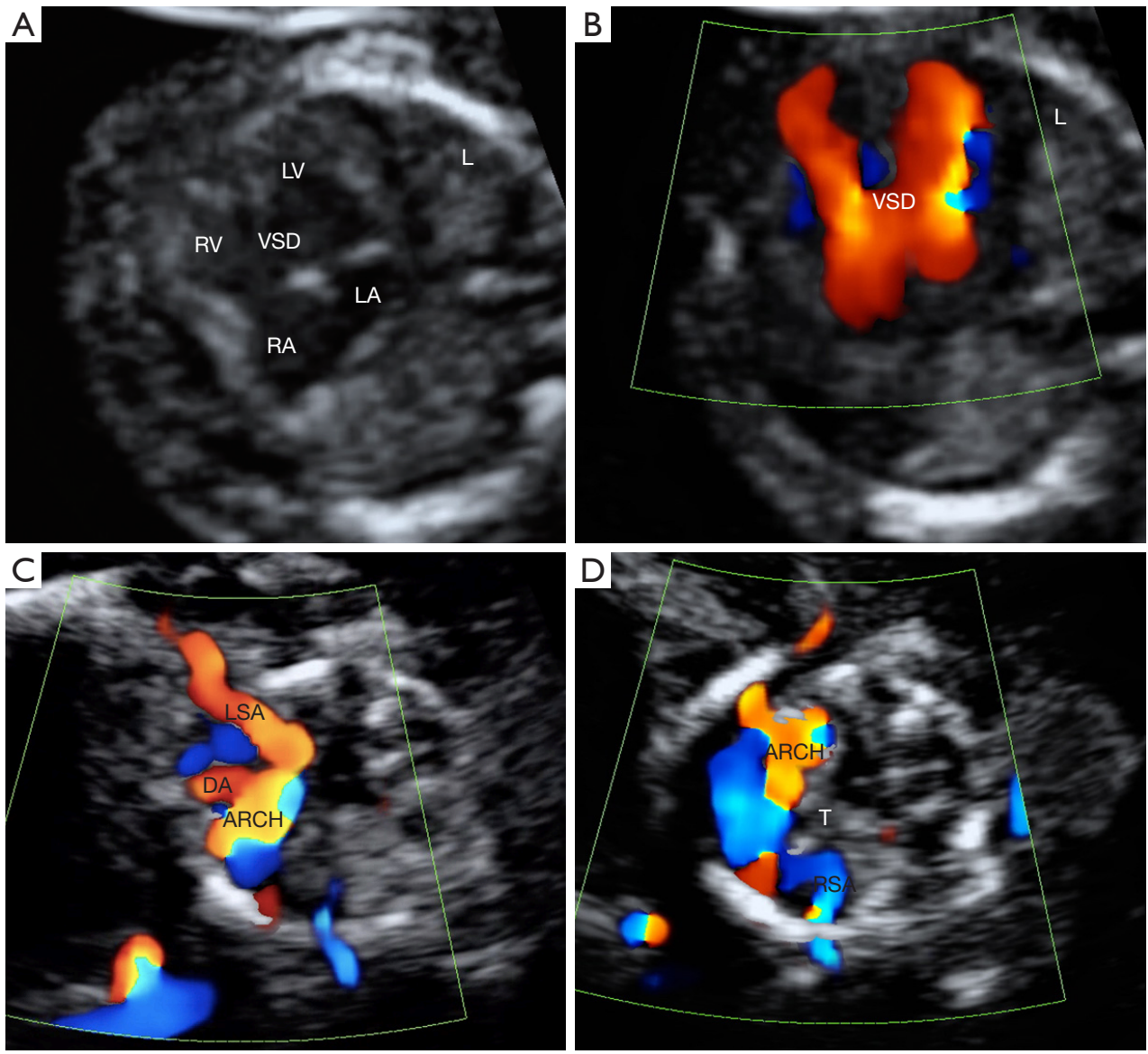

Figure 6 The 4-chamber heart and left and right subclavian arteries are clearly shown by TVUS. (A) VSD by TVUS; (B) TVUS showing VSD; (C) TVUS showing LSA; (D) TVUS showing RSA. TVUS, transvaginal ultrasound; VSD, ventricular septal defect; RV, right ventricle; LV, left ventricle; RA, right atrium; LA, left atrium; LSA, left subclavian artery; RSA, right subclavian artery; L, left; DA, arterial catheter; ARCH, arch aorta; $\mathrm{T}$, trachea.

than that of transabdominal ultrasonography, and the filling of blood flow by Doppler is also much higher than that of transabdominal ultrasonography, but this method depends more on fetal posture and requires examiners to have rich expertise professional knowledge and flexible operation level, and some women are reluctant to undergo transvaginal ultrasound examination (19). Based on the uncertainty of the above factors, the wide application of this technology is hindered.

A study of many patients by Ebrashy et al. (20) showed that the resolution of most fetal organs was improved by TVUS at 11-13 weeks, but there was no difference between transvaginal and transabdominal ultrasound in the contribution of CHD detection rate $(61.4 \%$ vs. $62.7 \%$; $\mathrm{P}=0.414)$. The results of this study are like ours. The results of statistical analysis showed that there was no significant difference between TVUS and transabdominal ultrasound, and they were consistent. However, transvaginal ultrasound provides a higher resolution, and transvaginal ultrasound can significantly reduce the misdiagnosis rate when the cardiac structure is suspected to be abnormal. Karim (21) and others believe that the use of transabdominal combined transvaginal ultrasound mode as needed is the only way to determine the real potential of early fetal malformations.

The deficiency of this study is that the sample size of chromosome karyotype analysis of fetuses with abnormal heart is small, mainly because early pregnancy screening gives parents a chance to choose pregnancy outcome earlier, pregnant women with CHD complicated with multiple extracardiac malformations will strive to end pregnancy as soon as possible, while our central chromosome karyotype analysis (amniocentesis) is carried out in 18 weeks, 


\section{Page 10 of 11}

choriocentesis at 13 weeks needs to be examined outside the province, and the waiting period is long. So many parents choose to terminate the pregnancy as soon as possible. In this study, half of the 66 cases of CHD chose induced labor in early pregnancy. Most of the fetal heart structures of pregnant women who underwent chromosome karyotype examination at 18 weeks were normal or with slight abnormalities, which could not fully reflect the chromosome distribution of CHD in early pregnancy.

\section{Acknowledgments}

Funding: None.

\section{Footnote}

Reporting Checklist: The authors have completed the STARD reporting checklist. Available at https://dx.doi. org/10.21037/atm-21-6492

Data Sharing Statement: Available at https://dx.doi. org/10.21037/atm-21-6492

Conflicts of Interest: All authors have completed the ICMJE uniform disclosure form (available at https://dx.doi. org/10.21037/atm-21-6492). The authors have no conflicts of interest to declare.

Ethical Statement: The authors are accountable for all aspects of the work in ensuring that questions related to the accuracy or integrity of any part of the work are appropriately investigated and resolved. All procedures performed in this study involving human participants were in accordance with the Declaration of Helsinki (as revised in 2013). The study was approved by Ningxia Medical University General Hospital scientific research Ethics Committee and informed consent was taken from all the mothers.

Open Access Statement: This is an Open Access article distributed in accordance with the Creative Commons Attribution-NonCommercial-NoDerivs 4.0 International License (CC BY-NC-ND 4.0), which permits the noncommercial replication and distribution of the article with the strict proviso that no changes or edits are made and the original work is properly cited (including links to both the formal publication through the relevant DOI and the license). See: https://creativecommons.org/licenses/by-nc-nd/4.0/.
Ji et al. Combined more soft indexes are valuable to forecast CHD

\section{References}

1. Hoffman JI, Christianson R. Congenital heart disease in a cohort of 19,502 births with long-term follow-up. Am J Cardiol 1978;42:641-7.

2. Yoon PW, Olney RS, Khoury MJ, et al. Contribution of birth defects and genetic diseases to pediatric hospitalizations. A population-based study. Arch Pediatr Adolesc Med 1997;151:1096-103.

3. Zhang J, Huang GY. Research progress on etiology and prevention of congenital heart disease. Chinese Journal of Evidence-Based Pediatrics 2012;7:231-8.

4. Copel JA, Cullen M, Green JJ, et al. The frequency of aneuploidy in prenatally diagnosed congenital heart disease: an indication for fetal karyotyping. Am J Obstet Gynecol 1988;158:409-13.

5. Schwanitz G, Zerres K, Gembruch U, et al. Prenatal detection of heart defects as an indication for chromosome analysis. Ann Genet 1990;33:79-83.

6. Eydoux P, Choiset A, Le Porrier N, et al. Chromosomal prenatal diagnosis: study of 936 cases of intrauterine abnormalities after ultrasound assessment. Prenat Diagn 1989;9:255-69.

7. Friedman AH, Copel JA, Kleinman CS. Fetal echocardiography and fetal cardiology: indications, diagnosis and management. Semin Perinatol 1993;17:76-88.

8. Crawford DC, Chita SK, Allan LD. Prenatal detection of congenital heart disease: factors affecting obstetric management and survival. Am J Obstet Gynecol 1988;159:352-6.

9. Souka AP, Krampl E, Bakalis S, et al. Outcome of pregnancy in chromosomally normal fetuses with increased nuchal translucency in the first trimester. Ultrasound Obstet Gynecol 2001;18:9-17.

10. Nicolaides KH. Nuchal translucency and other firsttrimester sonographic markers of chromosomal abnormalities. Am J Obstet Gynecol 2004;191:45-67.

11. Baś-Budecka E, Perenc M, Sieroszewski P. The role of fetal nuchal translucency (NT) and ductus venosus blood flow (DV) in the detection of congenital heart defects. Ginekol Pol 2010;81:272-6.

12. Ozkaya O, Sezik M, Ozbasar D, et al. Abnormal ductus venosus flow and tricuspid regurgitation at 11-14 weeks' gestation have high positive predictive values for increased risk in first-trimester combined screening test: results of a pilot study. Taiwan J Obstet Gynecol 2010;49:145-50.

13. Makrydimas G, Sotiriadis A, Ioannidis JP. Screening performance of first-trimester nuchal translucency for 
major cardiac defects: a meta-analysis. Am J Obstet Gynecol 2003;189:1330-5.

14. Carvalho JS, Moscoso G, Tekay A, et al. Clinical impact of first and early second trimester fetal echocardiography on high-risk pregnancies. Heart 2004;90:921-6.

15. Salomon LJ, Alfirevic Z, Bilardo CM, et al. ISUOG practice guidelines: performance of first-trimester fetal ultrasound scan. Ultrasound Obstet Gynecol 2013;41:102-13.

16. International Society of Ultrasound in Obstetrics and Gynecology, Carvalho JS, Allan LD, et al. ISUOG Practice Guidelines (updated): sonographic screening examination of the fetal heart. Ultrasound Obstet Gynecol 2013;41:348-59.

17. Sonek J. First trimester ultrasonography in screening and detection of fetal anomalies. Am J Med Genet C Semin Med Genet 2007;145C:45-61.

18. Geipel A, Gembruch U. Screening performance of first

Cite this article as: Ji X, Xia Y, Zhang H, Wang H, Shi R, Han Z. Study on the application of ultrasonography in the diagnosis of fetal cardiac structural abnormalities and the relationship between fetal cardiac structural abnormalities with chromosome abnormalities in early pregnancy. Ann Transl Med 2021;9(24):1790. doi: 10.21037/atm-21-6492 trimester nuchal translucency, ductus venosus blood flow and tricuspid regurgitation for cardiac defects. $\mathrm{Z}$ Geburtshilfe Neonatol 2012;216:157-61.

19. Abu-Rustum RS, Daou L, Abu-Rustum SE. Role of firsttrimester sonography in the diagnosis of aneuploidy and structural fetal anomalies. J Ultrasound Med 2010;29:1445-52.

20. Ebrashy A, El Kateb A, Momtaz M, et al. 13-14-week fetal anatomy scan: a 5-year prospective study. Ultrasound Obstet Gynecol 2010;35:292-6.

21. Karim JN, Roberts NW, Salomon LJ, et al. Systematic review of first-trimester ultrasound screening for detection of fetal structural anomalies and factors that affect screening performance. Ultrasound Obstet Gynecol 2017;50:429-41.

(English Language Editor: L. Huleatt) 